\title{
In Vivo Proton Magnetic Resonance Spectroscopy of the Brain in a Patient with L-2-Hydroxyglutaric Acidemia
}

\author{
FOLKER HANEFELD, BERND KRUSE, HARALD BRUHN, AND JENS FRAHM \\ Universitätskinderklinik, Schwerpunkt Neuropädiatrie, D-37070 Göttingen, Germany /F.H., B.K.]; and \\ Biomedizinische NMIR Forschungs GmbH, Max-Planck-Institut fur biophysikalische Chemie, \\ D-37018 Göttingen, Germany' [B.K., H.B., J.F.]
}

\begin{abstract}
Morphologic appearance and metabolic disturbances of the brain of a patient with L-2-hydroxyglutaric acidemia were investigated with use of magnetic resonance imaging and localized proton magnetic resonance spectroscopy in vivo. Whereas magnetic resonance imaging revealed increased internal and external cerebrospinal fluid spaces as well as patchy white matter lesions, metabolic deviations included a $\mathbf{5 0 \%}$ decrease of $\mathrm{N}$-acetylaspartate (neuronal marker), a $75 \%$ increase of myo-inositol (glial marker), and a $40 \%$ decrease of choline-containing compounds in white matter relative to age-matched controls. $\boldsymbol{A}$ clinical deterioration of the patient was clearly reflected in a follow-up examination 22 mo later, resulting in a further reduction of $\mathrm{N}$-acetylaspartate and a more pronounced enhancement of myo-inositol. No elevation of lactate was observed. The magnetic resonance spectroscopy findings are in line with a generalized neurodegenerative process in L-2-hydroxyglutaric acidemia but also suggest a defect in phosphatidyl inositol metabolism of glial cells. (Pediatr Res 35: 614-616, 1994)
\end{abstract}

\section{Abbreviations}

Cho, choline-containing compounds

$\mathrm{CSF}$, cerebrospinal fluid

Ins, inositol

L2OHglu, L-2-hydroxyglutaric

MRI, magnetic resonance imaging

MRS, magnetic resonance spectroscopy

NAA, $N$-acetylaspartate

$\mathrm{Cr}$, creatine and phosphocreatine

$\mathrm{T} 1$, spin-lattice relaxation time

$\mathrm{T} 2$, spin-spin relaxation time

L2OHglu acidemia is a rare inherited neurometabolic disease with eight cases including three pairs of siblings reported in Europe so far (1). The patients had cerebellar ataxia (seven of eight) and mild mental retardation (eight of eight) in combination with various other clinical and paraclinical signs such as extrapyramidal and pyramidal symptoms, seizures, and abnormalities on EEG. Biochemical findings included increased levels of L2OHglu acid in body fluids such as urine, plasma, and CSF, as

Received March 18, 1993; accepted December 6, 1993

Correspondence and reprint requests: Prof. Dr. Folker Hanefeld, Universitätskinderklinik, Schwerpunkt Neuropädiatrie, Robert-Koch-Str. 40, D-37070 Göttingen, Germany.

Supported by the Bundesminister für Forschung und Technologie of the Federal Republic of Germany (Grant 01 VF 8606/6) and by the Deutsche Forschungsgemeinschaft (B.K., Grant Kr 1337/1-1). well as a high CSF/plasma ratio, indicating intracerebral production. Lysine concentrations in CSF and plasma were also elevated, suggesting a relationship with the catabolism of lysine. A recent study of four more cases by Barth et al. (personal communication) discussed the possibility of a disturbed L-2-hydroxyacid-dehydrogenase function.

Here we report the findings of a combined MRI and localized proton MRS study of one of the patients with $\mathrm{L} 2 \mathrm{OHglu}$ acidemia described previously, i.e. patient 4 of the study by Barth $c t$ al. (1). The present results are part of an ongoing study of neurometabolic/neurodegenerative diseases that so far includes more than 80 cases with white matter diseases.

\section{MATERIALS AND METHODS}

Magnetic resonance studies. MRI/MRS examinations on two occasions 22 mo apart were performed after a mild sedation with midazolam on a 2.0-tesla MRI system (Siemens, Magnetom SP4000, Erlangen, Germany) with use of the standard imaging head coil for both MRI and MRS. After a fast-scan MRI protocol comprising T1-weighted fast low-angle shot images and T2weighted contrast-enhanced Fourier acquired steady state images (2), localized proton magnetic resonance spectra were acquired by means of a short echo time $(20 \mathrm{~ms})$ stimulated echo acquisition mode sequence with repetition times $3000 \mathrm{~ms}$ and 128 accumulations. Volumes of interest with sizes ranging from 8 to $18 \mathrm{~cm}^{3}$ were located in parietal white and gray matter and in the striatum. Technical details are given elsewhere (3). Quantitative spectral evaluations were based on a fully automated analysis of resonance areas with use of metabolite model spectra acquired under identical experimental conditions (4). Absolute concentration levels were calibrated with resonance areas obtained from reference solutions with known metabolite concentrations (5).

Patient. The female patient is the 1 st child of nonrelated healthy Turkish parents; one younger brother is healthy. Her mental and motor developments were only mildly delayed during the 1 st years. The 1st obvious symptoms occurred at the age of 2 y when she had a grand mal seizure. Furthermore, ataxia, spastic symptoms, and mild mental retardation were noticed. After a relatively stable period over many years, the clinical course of the disease showed a progressive deterioration since the age of $16 \mathrm{y}$. She now has repeated episodes of seizures and is unable to walk. Cranial computed tomography findings included white matter changes and enlarged CSF spaces. Visual evoked potentials at the age of 15 y showed prolonged latencies. Laboratory examinations yielded an increased $\mathrm{L} 2 \mathrm{OHglu}$ aciduria [193-832 $\mathrm{mmol} / \mathrm{mol}$ creatinine (normal, <2)], a mild hyperlysinuria in combination with a hyperlysinemia $(310-380 \mu \mathrm{mol} / \mathrm{L}$ (normal, <250)], and elevated CSF protein $(60-70 \mathrm{mg} / \mathrm{dL}$ ). $\mathrm{L} 2 \mathrm{OHglu}$ acid and lysine concentrations in CSF were not deter- 


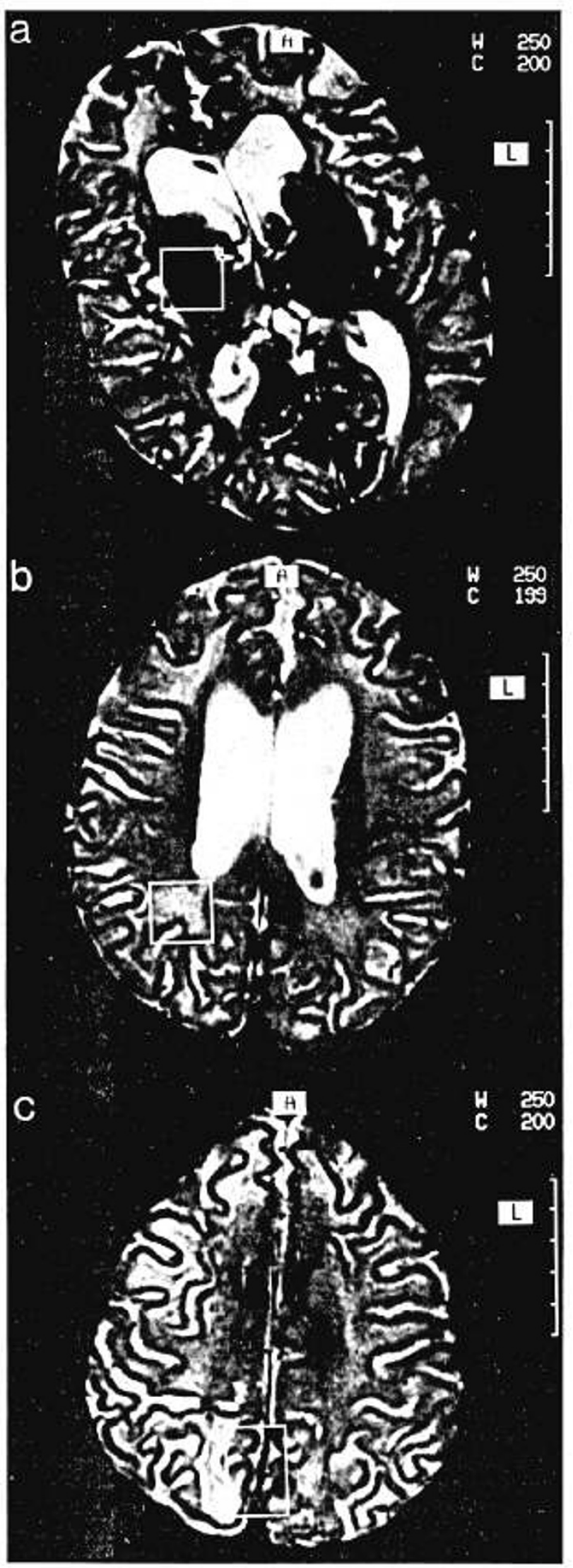

Fig. 1. White matter hyperintensities in transverse T2-weighted MRI sections (contrast-enhanced Fourier acquired steady state, repetition time/echo time $/ \alpha=14 /-6 / 40$ degrees, 4 -mm thickness) of a patient with $\mathrm{L} 2 \mathrm{OHglu}$ acidemia (first magnetic resonance examination at the age of $16 \mathrm{y}$ ). The boxes indicate volumes of interest selected for localized proton MRS as shown in Figure 2. Between acquisition of images $a$ and $b$, the patient was repositioned.
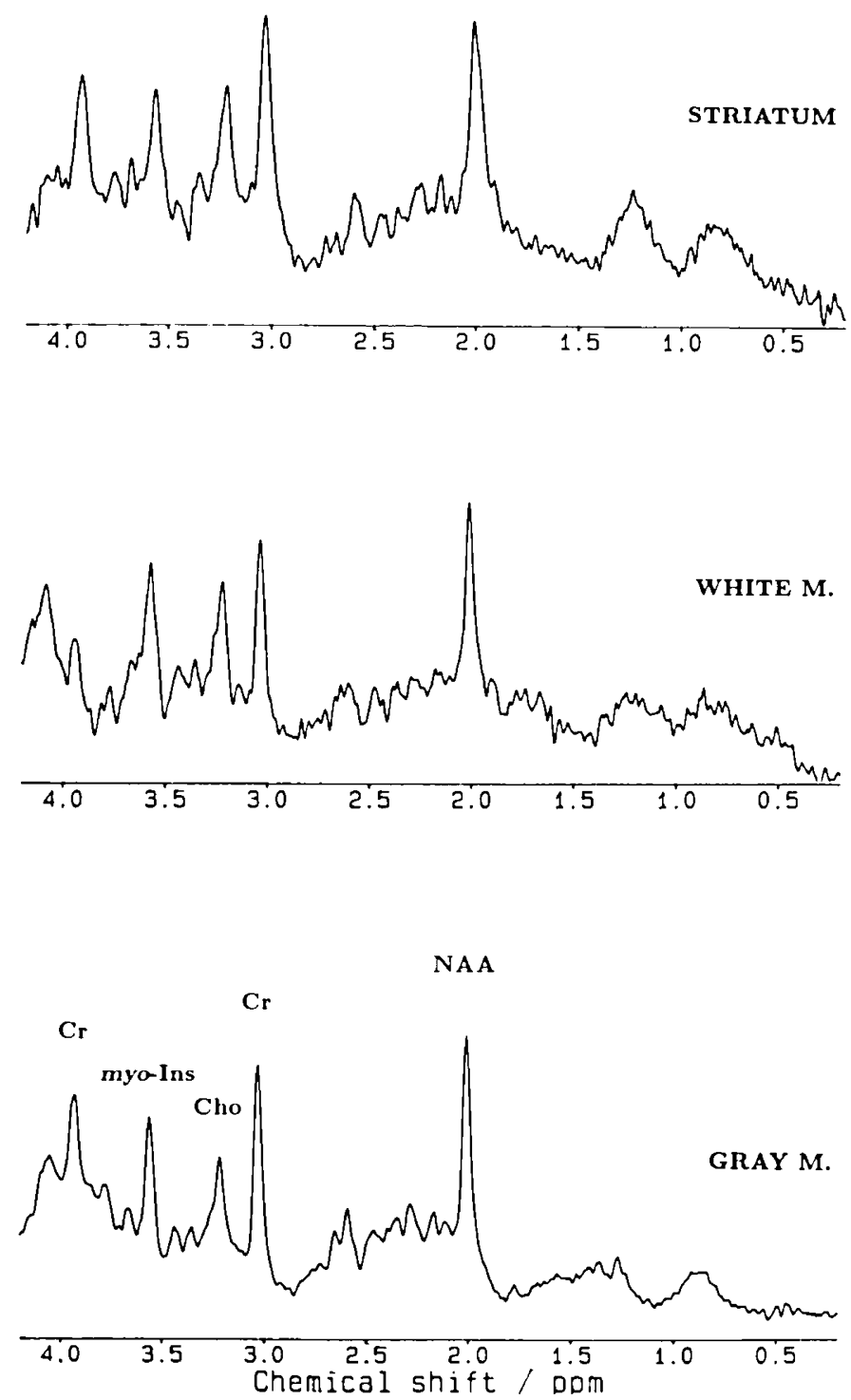

Fig. 2. Cerebral metabolic alterations in a patient with $\mathrm{L} 2 \mathrm{OHglu}$ acidemia (first magnetic resonance examination at the age of $16 \mathrm{y}$ ). Localized proton magnetic resonance spectra (stimulated echo acquisition mode (repetition time/echo time/middle interval $=3000 / 20 / 30 \mathrm{~ms}$, 128 accumulations) from the right striatum ( $8 \mathrm{~mL}$ volume of interest), right dorsoparietal paraventricular white matter $(8 \mathrm{~mL}$ volume of interest), and midline occipitoparietal gray matter ( $18 \mathrm{~mL}$ volume of interest) as indicated in Figure 1. Resonance frequencies (chemical shifts) from NAA, Cr, Cho, and mior-Ins are given in ppm and referenced to NAA $(2.01 \mathrm{ppm})$. All spectra have the same vertical and horizontal scale. Further resonances are caused by cytosolic proteins or short-chain fatty acids $(0.8-1.2 \mathrm{ppm})$, lactate (doublet at $1.33 \mathrm{ppm})$, glutamate $(2.1-2.3$ $\mathrm{ppm})$, scy/lo-inositol (3.35 ppm), and cerebral glucose (3.43 ppm).

mined. Details for laboratory procedures are discussed in Hoffmann el al. (6).

\section{RESULTS}

MRI at the age of $16 \mathrm{y}$ at the beginning of the patient's clinical deterioration revealed wide internal and external CSF spaces. In addition, prominent lesions in white matter were seen as hyperintense signals in T2-weighted images (Fig. 1) and as hypointensities in $\mathrm{T} 1$-weighted images of subcortical regions. White matter adjacent to the frontal and occipital horn of the lateral ventricles was similarly affected. No changes were noticed in the centrum semiovale. The boxes in Figure 1 indicate the positions of volumes of interest selected for MRS. 
Table 1. Cerebral metabolic changes*

\begin{tabular}{ccccc}
\hline & NAA & Cr & Cho & myo-Ins \\
\hline Patient & & & & \\
16 y old & 4.3 & 3.5 & 0.9 & 5.4 \\
18 y old & 2.4 & 3.5 & 0.8 & 6.0 \\
Controls & $8.37 \pm 0.76$ & $4.57 \pm 0.53$ & $1.45 \pm 0.15$ & $3.06 \pm 0.50$ \\
\hline
\end{tabular}

* Cerebral metabolic alterations in white matter of a patient with L2OHglu acidemia is age-matched controls $(n=10$; mean age, $14.5 \pm$ $2.8 \mathrm{y})$ as detected by quantitative evaluations of localized proton magnetic resonance spectra (repetition time/echo time/middle interval $=$ $3000 / 20 / 30 \mathrm{~ms}$ ). The values are proportional to absolute concentration levels (in $\mathrm{mM}$ ) without corrections for a mild TI saturation (repetition time $=3000 \mathrm{~ms}$ ) and for minor differential T2 losses (echo time $=20$ $\mathrm{ms}$, in vivo vs in vitro reference measurements).

The corresponding proton MRS examination showed marked disturbances in both gray and white matter as compared with metabolite patterns of healthy controls (Fig. 2). Major abnormalities included a $50 \%$ reduction of $\mathrm{NAA}$, a $40 \%$ reduction of Cho, and a $25 \%$ reduction of $\mathrm{Cr}$. In addition, white matter revealed an increase of the myo-Ins concentration by about $75 \%$. Although some of these findings may be recognized directly from the spectra (Fig. 2), quantitative metabolic changes for NAA, $\mathrm{Cr}$, Cho, and myo-Ins in white matter are more clearly appreciated from the values given in Table 1 in comparison to age-matched controls. The numbers are proportional to pertinent concentration levels (in $\mathrm{mM}$ ) but do not contain corrections for a mild T1 saturation (repetition time $=3000 \mathrm{~ms}$ ) and for minor differential $\mathrm{T} 2$ losses (echo time $=20 \mathrm{~ms}$, T2 differences between in vivo and in vitro). It should be noted that neither lysine nor $\mathrm{L} 2 \mathrm{OHglu}$ acid could be detected by proton MRS because of their low concentrations $(<1 \mathrm{mM})$.

The relevance of the observed metabolic disturbances was confirmed in a follow-up examination 22 mo later after a further clinical deterioration. Whereas MRI showed similar findings with slightly progressed atrophy as evidenced by increased CSF spaces. proton MRS of paraventricular white matter resulted in an even more dramatic decrease of NAA to about $20-30 \%$ of its normal level. As shown in Table 1, the concentration levels of $\mathrm{Cr}$ and Cho remained unchanged with respect to the first magnetic resonance examination, whereas myo-Ins was further increased to twice its normal value.

\section{DISCUSSION}

With regard to the exclusive location of NAA in neurons, its absence in glial tumors, and its decrease and irreversible loss in ischemic brain regions, this metabolite may be considered a marker for vital neuroaxonal tissue. Hence, its overall reduction in the brain of this patient with $\mathrm{L} 2 \mathrm{OHglu}$ acidemia provides a measure of progressive neuroaxonal degeneration. The absence of elevated lactate levels at both examinations indicated undisturbed mitochondrial respiration. An interesting and potentially specific finding is the opposite variation of Cho and myo-Ins. So far, increased myo-Ins has only been observed in conjunction with elevated Cho in diseases such as gliomas (7), multiple sclerosis (8), and metachromatic leukodystrophy (9), where the concomitant increase of both metabolites is thought to mark membranoproliferative processes and glial growth. In contrast, the present findings more likely reflect an inhibition of the synthesis of phosphatidyl inositol in glial cells that leads to an accumulation of the precursor myo-Ins. The extent of Cho reduction then fits into the generalized neurodegenerative process that commonly characterizes progressed stages of inherited metabolic diseases.

\section{REFERENCES}

1. Barth PG, Hoffmann GF, Jacken J, Lehnert W, Hanefeld F, van Gennip AH, Duran M, Valk J, Schutgens RBH, Trefz FK, Reimann G, Hartung HP 1992 L.-2-Hydroxyglutaric acidemia: a novel inherited neurometabolic disease. Ann Neurol 32:66-7!

2. Frahm J, Gyngell ML. Hänicke W 1991 Rapid scan techniques. In: Stark DD, Bradley WG (eds) Magnetic Resonance Imaging. CV Mosby, St. Louis, pp 165-203

3. Frahm J, Michaelis T. Merboldt KD, Bruhn H, Gyngell ML, Hänicke W 1990 Improvements in localized 'H-NMR spectroscopy of human brain. Water suppression, short echo times, and $1 \mathrm{~mL}$ resolution. J Magn Reson 90:464473

4. Provencher S, Michaelis T, Hänicke W, Frahm J 1992 Automated determination of metabolite concentrations from localized in vivo proton NMR spectra. Society of Magnetic Resonance in Medicine, 11 th Annual Meeting. Berlin, Germany, p 670 (abstr)

5. Michaelis T, Merboldt KD. Bruhn H, Hänicke W. Frahm J 1993 Absolute concentrations of metabolites in the adult human brain in vivo. Radiology 187:219-227

6. Hoffman GF, Voss W, Hunneman DH, Hamann J, Lehnert W, Jacobs C Hanefeld F 1990 L-2-Hydroxyglutarazidurie: Eine neue Enzephalopathie mit leukodystrophen Veränderungen. In: Hanefeld F, Rating D, Christen HJ (eds) Aktuelle Neuropädiatrie, Springer-Verlag, Berlin Heidelberg, Germany, pp 139-142

7. Frahm J, Bruhn II, Merboldt KD, Hänicke W, Mursch K, Markakis E 1991 Localized proton NMR spectroscopy of brain tumors. Methodologic improvements using short-echo time STEAM sequences. J Comput Assist Tomogr 15:915-922

8. Bruhn H, Frahm J, Merboldt KD. Hänicke W, Christen HJ, Kruse B, Hanefeld F, Bauer, HJ 1992 Multiple sclerosis in children. Cerebral metabolic alterations monitored by localized proton magnetic resonance spectroscopy in vivo. Ann Neurol 32:140-150

9. Kruse B, Hanefeld F, Christen HJ, Bruhn H, Michaelis T, Hänicke W, Frahm J 1993 Alterations of brain metabolites in metachromatic leukodystrophy as detected by localized proton MR spectroscopy in vivo. J Neurol 241:68-74 